\title{
A success story in pharmacogenomics: genetic ID card for SJS/TEN
}

\author{
"The best solution for resource-rich countries would be to phase out \\ these culprit drugs."
} First draft submitted: 14 December 2015; Accepted for publication: 14 December 2015;
Published online: 30 March 2016

Keywords: clinical implementation • counseling • PGx Card • pharmacogenomics • SJS/TEN

Stevens-Johnson syndrome (SJS) and toxic epidermal necrolysis (TEN) are severe mucocutaneous reactions, commonly triggered by medicines, characterized by extensive necrosis and detachment of the epidermis. Though, the genetically mediated SJS/TEN are rare and only induced by certain drugs, and only in people with specific risk alleles of immune genes. However, the damage is so extensive that patients typically end up in burn care facilities, in excruciating pain, unable to eat, urinate or open their eyes. Skin starts peeling off in sheets from large areas of the body. SJS is a minor form of toxic epidermal necrolysis, with $<10 \%$ body surface area (BSA) detachment. Overlapping SJS/TEN: detachment of $10-30 \%$ of the BSA. TEN: detachment of $>30 \%$ of the BSA. It is so painful that many patients want to die; some do [1].

Astonishingly, to deal with it, we do not need to edit genes, turn to stem cells or gene therapy, prescribing drugs that target mutations in a patient's genome. We simply screen people for the risky variants and withhold the triggering drugs. It is 'the low-hanging fruit of genomic medicine'. In January 2014, while scientists from resource-rich countries were discussing about whole-genome sequencing for millions of their people, to the delegates at the Global Leaders in Genomic Medicine Summit, a multinational coalition of genomic and policy experts from over 20 countries working to implement genomics in clinical care organized by the National Human Genome Research Institute and NIH - The
Global Genomic Medicine Collaborative [2], a simple wallet pharmacogenetic plastic card from Thailand, a resource-limited setting, was first presented to those members. Surprisingly, they most agreed that pharmacogenetic card system was a real opportunity, not only a genetic finding but a way of implementing it. They wanted to have the pharmacogenetic ID card implemented in their resource-rich countries as well.

In 2011, Dr C Sukasem at Bangkok's Ramathibodi Hospital invented this low-tech approach. After each patient gets the pharmacogenetic tests, their results are entered into the 'pharmacogenetic ID card', a purple rectangle and wallet size that they carry around and show to future doctors (Figure 1). It is simple, cost effective, and although it is still being formally evaluated, all indications suggest that it is working. After the Global Genomic Medicine Collaborative meeting in 2014, National Human Genome Research Institute/NIH quickly organized a 2-day meeting in March 2015 to discuss research priorities for SJS/TEN, and how to monitor or manage 'serious' and 'severe' cutaneous adverse drug reactions more broadly. Pharmacogenetic ID card was one of the main topics that were discussed.

The first pharmacogenetic screening test for SJS/TEN was started in Asia in 2004. Chung and colleagues found the strong association between the $H L A-B^{*} 15: 02$ allele and carbamazepine-induced SJS/TEN by sequencing HLA genes from patients [3]. Later, the

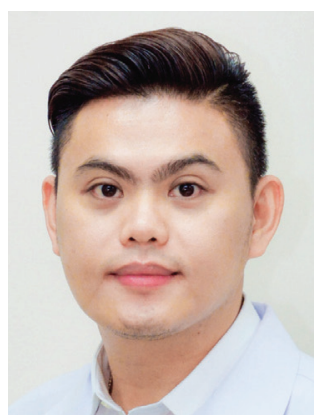

Chonlaphat Sukasem

Division of Pharmacogenomics \& Personalized Medicine, Department of Pathology, Faculty of Medicine Ramathibodi Hospital, Mahidol University, Bangkok, Thailand and

Laboratory for Pharmacogenomics, Somdech Phra Debaratana Medical Center (SDMC), Faculty of Medicine Ramathibodi Hospital, Bangkok, Thailand

\section{Wasun Chantratita Author for correspondence: Virology Laboratory, Department of Pathology, Faculty of Medicine, Ramathibodi Hospital, Mahidol University, Bangkok, Thailand and \\ Center for Medical Genomics, Ramathibodi Hospital, Mahidol University, Bangkok, Thailand Tel.: + 6622011470 Fax: + 6622011470 wasun.cha@mahidol.ac.th}



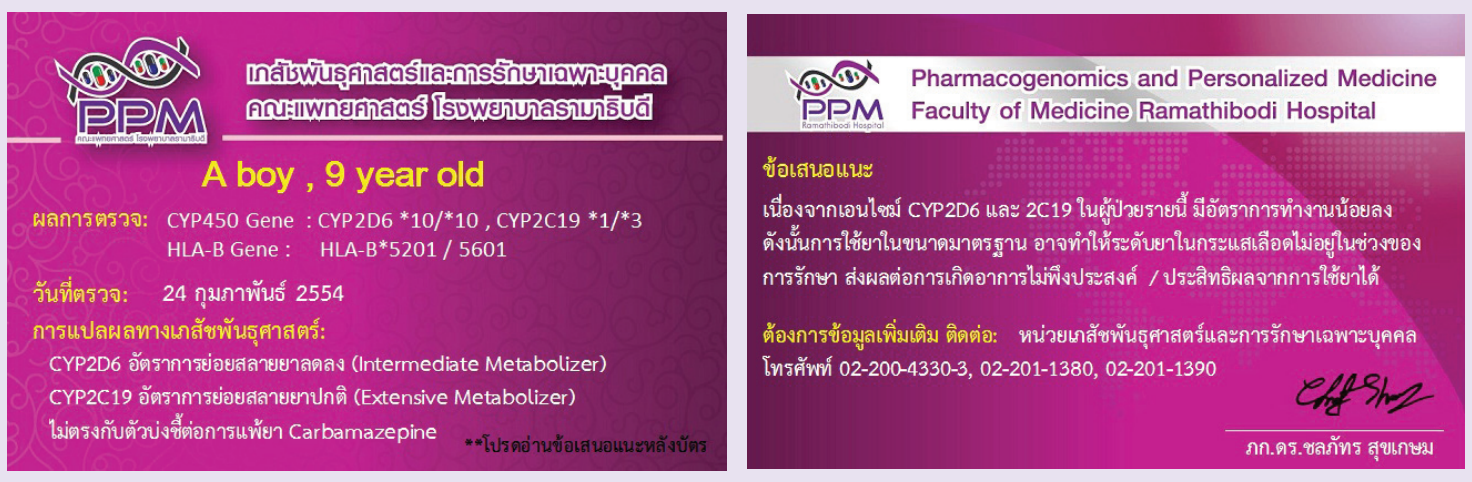

Figure 1. Pharmacogenetic ID card of a boy, 9 years old with ASD in Thailand. His physician would like to prescribe carbamazepine and risperidone for treatment. The pharmacogenetic tests (ASD all-in-one assay; HLA-B, CYP2D6 and CYP2C19) were ordered after pre-pharmacogenetic test counseling. After genotyped and interpreted, the patients' genetic were then resulted and entered into the pharmacogenetic ID card. The post-pharmacogenetic test counseling was eventually advised on drug selection and dose adjustment by a trained pharmacogeneticain. ASD: Autism spectrum disorder.

$H L A-B^{*} 58: 01$ allele, with allopurinol-induced SJS/ TEN, was found in the Taiwanese and southeast Asian population [4]. Other scientists soon identified many other drugs that induce severe cutaneous adverse reaction syndromes, including anti-HIV medications such as nevirapine [5], abacavir [6], stavudine [7], and anticonvulsants like phenytoin [8]. Each drug is associated with its own particular risks in $H L A$ variants.

“...pre- and post-pharmacogenetic test counseling may be the most necessary for clinical pharmacogenomics implementation."

After extensive economic evaluations for the reduction of national healthcare expenditure, Taiwan's national health insurance covered the cost of the PCR-based pharmacogenetic assay for $H L A-B^{*} 15: 02$ in 2010 [9]. The Thai universal health coverage scheme has done the same since 2012 [10] to reimburse the cost of $\$ 28$ per person. This pharmacogenetic testing has worked effectively. Ten years ago, 10-20 people were admitted into Taiwanese hospitals yearly with SJS/TEN, but today there are only a few of annual cases [11]. In Thailand, the incidence of SJS/ TEN has reduced sharply [Manolio ta. Research direcTIONS in GENETICALly MEDiATED STEVENS-JoHNSON SYNDROME/ TOXIC EPIDERMAL NeCROLYSIS (2015). MANUSCIPT IN PREPARATION], and now the country is in the phase of eradicating this life-threatening severe cutaneous adverse drug reaction.

Does this means that SJS/TEN could be quickly reduced and eradicated? Focus screening of four prevalent risk alleles; $H L A-B^{*} 15: 02, H L A-B^{*} 58: 01$, $H L A-B^{*} 57: 01$ and $H L A-B^{*} 35: 05$ variants, before high risk populations starting carbamazepine [3], allopuri- nol [4], abacavir [6] and nevirapine [5] therapy, respectively, can it be significantly averted occurrence of SJS/TEN globally? The answer might be 'No'. Though, the easiest way to lower the incidence of SJS/TEN, of cause would be to screen people for risky variants and withhold triggering drugs, even prior to drug approval and release. However, it would be harder to implement low-cost pharmacogenetic assay(s) in state/national health programs, with the hardest part being safe and effective utilization of pharmacogenomics in the hospital.

The best solution for resource-rich countries would be to phase out these culprit drugs. In Taiwan, carbamazepine prescriptions have fallen by nearly $70 \%$ in the last decade [12], as doctors have changed to a safer second generation of antiepileptics. However, since these newer drugs are more expensive, some resourcelimited countries, like Thailand, have found it harder to make the switch. It is more cost effective for patients in Thailand, who are due to receive carbamazepine therapy, to be screened for the genetic marker before receiving therapy. Universal $H L A-B^{*} 15: 02$ screening indicates good value for money in terms of preventing SJS/TEN in carbamazepine-treated patients in the developing country, Thailand [10]. With the use of pharmacogenetic screening for genetic variants, the potential of serious adverse events can be avoided.

To strengthen the utilization of pharmacogenetic testing in hospital, Dr Chonlaphat Sukasem at Bangkok's Ramathibodi Hospital has first invented and launched a pharmacogenetic card approach (Figure 1). After patients have taken an HLA test, their results are entered into a plastic 'pharmacogenetic wallet card', which contains the genomic results, such as HLA genotyping outcome of those related to the 
risk of SJS/TEN [13]. This card can be carried around and shown to various doctors in the future. In order to serve the needs of patients, such pharmacogenetic cards recently have been expanded to more drug/ pharmacogenetic biomarkers, such as $H L A-B$ and CYP450 genes depending on the medication used, which is simple and cost effective. Pharmacogenetic testing of $H L A-B^{*} 5701, H L A-B^{*} 3505, H L A-B^{*} 4001$ and $C Y P 2 B 6$ implemented along with a pharmacogenetic card before patients took antiretroviral drugs, abacavir, nevirapine, stavudine and efavirenz [13]. Recently, the treatment planning was after the physicians were informed about the results of pharmacogenetics testing. Kiertiburanakul and her colleague showed that among 27 antiretroviral treated in Thai HIV-infected patients, abnormal laboratory results, such as renal insufficiency from tenofovir or anemia from zidovudine, were improved and some returned to normal in 59.3\%. HIV RNA was undetectable after treatment adjustment in $94.9 \%$ [13].

The genetic variants associated most commonly with SJS/TEN are usually found in people of Asian descent. For example, $H L A-B^{*} 15: 02$ is found in up to $8 \%$ of people in Taiwan, Thailand and other parts of southeast Asia, but is rare in Japan, Korea and Europe [14]. However, SJS/TEN is still a global problem. Western countries have their own deadly combinations of drugs and HLA variants. Phillips et al. showed that people with the $H L A-B^{*} 57: 01$ variant can develop SJS/TEN after taking abacavir [9]. That variant is common in Europe and has become the subject of routine testing largely because abacavir is used specifically to treat HIV.

Anyhow, SJS/TEN may not disappear easily, due to human error. A clinician might ask a patient to have a blood test, but fail to file the results. Then other doctors cannot see this information, and could prescribe a lethal drug. An electronic-health system that automatically notifies doctors and pharmacists of a patient's test results would solve this problem, with a warning sign popping up in an electronic medical record (EMR). Most SJS/TEN-inducing medications are used broadly. It is virtually impossible for all physicians to remember the details of SJS/TEN-inducing medications without prompts and decision supports from the EMR.

In addition, not only pharmacogenetics testing equipped with pharmacogenetic ID card alone, but also these following factors below play important roles in the success of reducing and hopefully eradicating genetically induced SJS/TEN. These factors include available public and clinician awareness of pharmacogenomics in individual countries, a robust and effective pharmacovigilance system, the pharmaco- genomics laboratory and knowledge of genetic interpretation, alternative drugs, turnaround time of tests, and tablet or smartphone applications for educating patients and the general public on genomic medicinesevere adverse drug reaction. Additionally, pre- and post-pharmacogenetic test counseling may be the most necessary for clinical pharmacogenomics implementation. In 2011, the pharmacogenomics clinic has been officially operated for pre- and post-pharmacogenetic test counseling by a trained pharmacogenetician (C Sukasem), the combined pharmacy knowledge and pharmacogenetics skill. The counseling system is not just only delivered by the clinical appointment for face-to-face counseling but it can also be provided by various approaches such as telephone, e-mail and bedside counseling to communicate results to admitted patients for proof of 'from bench to bed-side concept' in Thailand. The pharmacogenetic counselor can play an important role in delivering pharmacogenetic testing by informing and assisting both health practitioners and patients in the interpretation and communication of genetic information as an educational resource and advising on drug selection, dose adjustment, and therapeutic drug monitoring based on the pharmacogenetic results and other clinical factors such as drug interaction.

In summary, we recommended at least seven primary requirements to succeed the pharmacogenomics in clinical implementation: pharmacogenetics tools (pharmacogenetic card, precision medicine report and interpretation systems); pharmacogenetics infrastructure and facilities (pharmacogenomics laboratory, pharmacogenomics clinic and pharmacogenetic testing); pre- and post-pharmacogenetics counseling; pharmacogenetic knowledge and education for health professionals; pharmacovigilance system; EMR and training program for the pharmacist to be a pharmacogenetician.

\section{Financial \& competing interests disclosure}

The authors would like to thank the Center for Medical Genomics, Ramathibodi Hospital, Mahidol University, Bangkok, Thailand; Thailand Center of Excellence for Life Sciences (TCELS), Ministry of Science and Technology; The Khoon Poom Foundation, The Project of Her Royal Highness Princess Ubonratana Rajakanya Siriwatana Bhanawadee; Pharmacogenomics for Autistic Children, Office of National Research Council of Thailand. The authors have no other relevant affiliations or financial involvement with any organization or entity with a financial interest in or financial conflict with the subject matter or materials discussed in the manuscript apart from those disclosed.

No writing assistance was utilized in the production of this manuscript. 


\section{References}

1 Sukasem C, Puangpetch A, Medhasi S et al. Pharmacogenomics of drug-induced hypersensitivity reactions: challenges, opportunities and clinical implementation. Asian Pac. J. Allergy Immunol. 32, 111-123 (2014).

2 Manolio TA, Abramowicz M, Al-Mulla F et al. Global implementation of genomic medicine: we are not alone. Sci. Transl. Med. 7, 290ps13 (2015).

3 Chung WH, Hung SI, Hong HS et al. Medical genetics: a marker for Stevens-Johnson syndrome. Nature 428, 486 (2004).

4 Hung SI, Chung WH, Liou LB et al. HLA-B*5801 allele as a genetic marker for severe cutaneous adverse reactions caused by allopurinol. Proc. Natl Acad. Sci. USA 102, 4134-4139 (2005).

5 Chantarangsu S, Mushiroda T, Mahasirimongkol S et al. $H L A-B^{*} 3505$ allele is a strong predictor for nevirapineinduced skin adverse drug reactions in HIV-infected Thai patients. Pharmacogenet. Genomics 19, 139-146 (2009).

6 Lucas A, Nolan D, Mallal S. HLA-B*5701 screening for susceptibility to abacavir hypersensitivity. J. Antimicrob. Chemother. 59, 591-593 (2007).

7 Wangsomboonsiri W, Mahasirimongkol S, Chantarangsu S et al. Association between $H L A-B^{*} 4001$ and lipodystrophy among HIV-infected patients from Thailand who received a stavudine-containing antiretroviral regimen. Clin. Infect. Dis. 50, 597-604 (2010)

8 Min FL, Shi YW, Liu XR et al. HLA-B*1502 genotyping in two Chinese patients with phenytoin-induced StevensJohnson syndrome. Epilepsy Behav. 20, 390-391 (2011).

9 Phillips EJ, Chung WH, Mockenhaupt M et al. Drug hypersensitivity: pharmacogenetics and clinical syndromes. J. Allergy Clin. Immunol. 127, S60-S66 (2011).

10 Rattanavipapong W, Koopitakkajorn T, Praditsitthikorn $\mathrm{N}$ et al. Economic evaluation of $H L A-B^{*} 15,02$ screening for carbamazepine-induced severe adverse drug reactions in Thailand. Epilepsia 54, 1628-1238 (2013).

11 Mitropoulos K, Al Jaibeji H, Forero DA et al. Success stories in genomic medicine from resource-limited countries. Hum. Genomics 9, 11 (2015).

12 Hsieh LP, Huang CY. Antiepileptic drug utilization in Taiwan: analysis of prescription using National Health Insurance database. Epilepsy Res. 84, 21-27 (2009).

13 Bushyakanist A, Puangpetch A, Sukasem C et al. The use of pharmacogenetics in clinical practice for the treatment of individuals with HIV infection in Thailand. Pharmgenomics Pers. Med. 8, 163-170 (2015).

14 Lee MT, Mahasirimongkol S, Zhang Y et al. Clinical application of pharmacogenomics: the example of HLAbased drug-induced toxicity. Public Health Genomics 17, 248-255 (2014). 